\title{
Mathematical structure of a bilevel strategic pricing model
}

\author{
Patrice Marcotte (corresponding author) • Gilles Savard • Daoli Zhu \\ DIRO and CRT, Université de Montréal \\ C.P. 6128, Succursale Centre-Ville \\ Montréal (Québec) Canada H3C 3J7 \\ MAGI and GERAD, École Polytechnique-Montréal \\ C.P. 6079, Succursale Centre-ville \\ Montréal (Québec) Canada HЗC 3A7 \\ Dept. of Management Science \\ Fudan University \\ 220 Handan Road, Shanghai 20433, China \\ marcotte@iro.umontreal.ca•gilles@crt.umontreal.ca・dlzhu@fudan.edu.cn
}

\begin{abstract}
This paper is concerned with the characterization of optimal strategies for a service firm acting in an oligopolistic environment. The decision problem is formulated as a leader-follower game played on a transportation network, where the leader firm selects a revenue-maximizing price schedule that takes explicitly into account the rational behavior of the customers. In the context of our analysis, the follower's problem is associated with a competitive network market involving non atomic customer groups. The resulting bilevel model can therefore be viewed as a model of product differentiation subject to structural network constraints.
\end{abstract}

Keywords: Pricing, Productivity and competitiveness, Revenue management, Economics, Game theory

\section{Introduction}

In the context of deregulation, much attention is being paid to the design of managerial tools for optimizing revenues. In the airline industry, a 'Yield Management' strategy (see McGill and Van Ryzin 1999, Belobaba 1987) consists in addressing, concurrently or sequentially, four interrelated issues: demand forecasting, pricing, seat allocation and overbooking. While an optimization model that deals simultaneously with these issues cannot be realistically considered, several attempts have been made at addressing them individually, one market at a 
time. However, the strong interconnections between markets, as well as the bilateral impacts of pricing and seat allocation policies, which are often designed by separate departments within an airline, point to the weaknesses of such disaggregate approaches.

In this paper, we consider a bilevel model of revenue management for an industry operating within an oligopolistic environment characterized by a network structure and the partition of demand into user groups, each group being endowed with its valuation of the attributes. Although the model could be applied to various sectors of an economy (hotels, manufacturing industry, etc.), we use as our base paradigm that of an airline whose aim is to design a revenue maximizing strategy based on jointly optimal pricing and seat allocation policies. Such a strategy takes explicitly into account the finite capacity of the leader's fleet, as well as the behaviour of each user group. This results in a strategy that discriminates between customers, according to their willingness to pay for flights with high quality of service or short duration. While we assume that the overall demand for transportation is inelastic over the planning horizon, the demand specific to the leader airline is dependent on its fare and seat allocation policies; in that sense, the model addresses, partially, the issue of demand forecasting previously mentioned as one of the four key components of the 'Yield Management' decision process.

Two of the model's features have been widely studied in the economic literature (see Martin 1993), namely product differentiation (Hotelling 1929, Gabszewicz and Thisse 1986) and customer segmentation (Gabszewicz and Thisse 1979, Phlips and Thisse 1983), both these features paving the way to price discrimination (Phlips 1983). The main goal of this paper is to characterize the market structure resulting from the underlying network topology, a result previously achieved only on a simple two-node network by Leurent (1993). Our approach can also be viewed as a differential markets extension of the taxation model of Labbé, Marcotte and Savard (1998).

Finally, the reader is referred to Loridan and Morgan (1996) for an analysis of the relationship between bilevel programs and Stackelberg (leader-follower) games.

The paper is structured as follows: in Section 2 we motivate and formulate a strategic model of revenue management, whose mathematical properties are analyzed in Section 3, and for which an algorithmic framework is developed Section 4. 


\section{A product differentiation model}

To illustrate the concepts introduced and analyzed in this paper, let us consider a system composed of airlines (or alliances) competing for market share on overlapping networks. Such a system, corresponding to the competitive market 'Montreal - Shanghai', is shown in Figure 1.

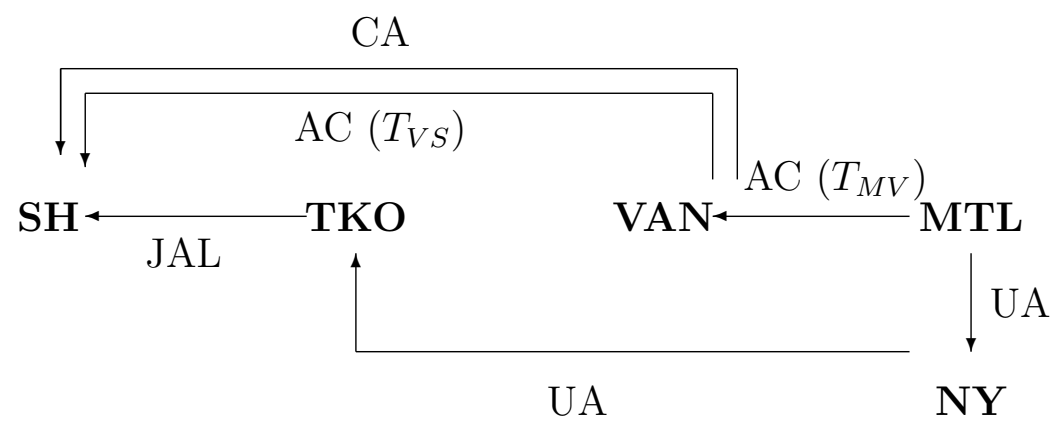

Figure 1: Structure of the flight market

In this network, the cities of Vancouver, Tokyo and New York correspond to connection hubs, while the arcs represent the various flight legs. In this example, we select $\mathrm{AC}$ as the leader company, which operates both a single leg flight to Vancouver and a connecting flight to Shanghai, and set the travellers to be the followers. This company selects fares $T_{M V}$ on the Montreal-Vancouver part of the trip and $T_{V S}$ on the transpacific leg, given fixed fare schedules from its competitors. For ease of presentation, we assume that fares are associated with legs although, in real life, they are associated with flights between origin and destination nodes (see Côté et al. 2002 for the description of a flight-based model). The flight structure offered by three competing companies over the Montreal - Shanghai market is specified in Table 1.

Flight Airline Path Rate Travel time

$1 \quad$ UA+JAL Montreal-New York-Tokyo-Shanghai $\$ 1320 \quad 36$ hours

2 AC+CA Montreal-Vancouver-Shanghai $\quad T_{M V}+\$ 720 \quad 26$ hours

$3 \quad \mathrm{AC}+\mathrm{AC} \quad$ Montreal-Vancouver-Shanghai $\quad T_{M V}+T_{V S} 18$ hours

Table 1: Flight structure for the Montreal - Shanghai market (prices in $\$ C A N$ ) 
The revenue of $\mathrm{AC}$ is clearly dependent on the fare structure within the system and on the demand for the entire flight composed of two legs. The demand for a given flight may be interpreted as the reaction to the market's fare structure and the attractiveness of the flight with respect to competing proposals. In the above simplistic example, flights are only differentiated by their respective duration and number of legs (i.e., number of connections plus one). In real life, passengers select a product endowed with several attributes. While price and time are the prime components of a given product, others have to be considered as well, such a the choice of a cabin (economy, business or first class) and of various features associated with a ticket (refundable or not, advance purchase, minimum stay, etc.). This results in as many as 25 products ('Fare Basis Codes') for a given flight, for which a number of aircraft seats has to be assigned. For notational simplicity, we shall not consider this seat allocation problem, and assume that all seats of the aircraft share the same attributes.

On the demand side, we assume that each traveller is characterized by a trade-off value between time and money, which we denote by the letter $\alpha$. If $T_{a}$ and $C_{a}$ are, respectively, the fare and the travel time associated with leg $a$ of some flight, then the perceived disutility (cost) $G C_{a}$ of a traveller of the 'group' having VOT (Value of Time) $\alpha$ is

$$
G C_{a}=T_{a}+\alpha C_{a}
$$

where $\alpha$ is a continuous random variable with density $\psi_{w}(\alpha)$ that could depend on the origindestination $w$. Natural and important extensions of this concept, which are not investigated in this paper, are nonlinear valuations of time and market segmentation of the VOT.

Faced with a supply for transportation from its origin node to its destination node, a customer selects the flight that minimizes its own disutility. Due to the continuity assumption on the distribution of $\alpha$, the choice of the optimal flight is nonambiguous, with the exception of a finite number of values of the parameter $\alpha$, whenever travel times for a given O-D pair are distinct (see Marcotte and Zhu 1997). The demand for a given flight is obtained by integrating the density function $\psi_{w}$ over the set of $\alpha$ values (intervals, actually) for which that flight is optimal. This yields a continuous and piecewise differential demand mapping.

Even on a problem of very small size, as that considered above, the choice of revenuemaximizing fares $T_{M V}$ and $T_{V S}$ is far from obvious, as it depends on

- the shape of the density function $\psi_{w}$, 
- the network structure (a high value of $T_{M V}$ might make sense on the Montreal-Vancouver market but deter customers headed for China),

- the attributes (fare and duration) of the competing flights.

The algorithmic issues related to the above three points will be addressed in Section 4 .

The above example, where airline flights correspond to 'products', is a case of horizontal product differentiation (see Krouse 1990) that takes place on a transportation network, hence a nontrivial structure, and where a service is realized by a sequence of 'legs' that make up a path of the network, i.e., a 'flight'. Before giving a formal statement of the mathematical model, we set the notation, in the context of an airline network.

$$
\begin{aligned}
G=(N, A) & \text { service network } \\
N & \text { set of nodes (origins, destinations, connecting nodes) of } G \\
A \subset N \times N & \text { set of arcs (legs) of } G \\
A_{1} \subset A & \text { set of arcs (legs) controlled by the leader } \\
A_{2}=A \backslash A_{1} & \text { set of arcs controlled by the competition } \\
W \subset N \times N & \text { set of origin-destination pairs (flights) } \\
K_{w} & \text { set of flights (network paths) connecting the origin-destination pair } w \in W \\
d_{w} & \text { demand associated with an origin-destination } w \in W \\
C_{a} & \text { flight duration on leg } a \\
T_{a}, a \in A_{2} & \text { fare on leg } a \text { (competition) } \\
\psi_{w}(\alpha) & \text { density of VOT parameter } \alpha \text { for origin-destination pair } w \in W \\
\alpha_{\max } & \text { maximum value for the VOT parameter. }
\end{aligned}
$$

The decision variables for both the leader and the followers are:

$$
\begin{aligned}
x_{a}(\alpha) & \text { flow density }(\operatorname{group} \alpha) \text { on leg } a \\
x_{a} & \text { total flow on } \operatorname{leg} a: x_{a}=\int_{0}^{\alpha_{\max }} x_{a}(\alpha) d \alpha \\
h_{k}(\alpha) & \text { flow density (group } \alpha) \text { on path } k \\
h_{k} & \text { total flow on path } k: h_{k}=\int_{0}^{\alpha_{\max }} h_{k}(\alpha) d \alpha \\
T_{a}, a \in A_{1} & \text { fare on leg } a \text { (leader) }
\end{aligned}
$$

In this system, the demand side consists of nonatomic consumers differentiated by their respective VOT parameter $\alpha$. The supply side consists of products/services that are relatively close substitutes to each other, and are represented by paths in the transportation network. Consumers are assumed to make indivisible and mutually exclusive purchases of the products or services.

The origin to destination (O-D) demands give rise to arc flows $x_{a}(\alpha), a \in A$ and the total generalized trip cost is

$$
\int_{0}^{\infty} \sum_{a \in A}\left(T_{a}+\alpha C_{a}\right) x_{a}(\alpha) d \alpha
$$


For each origin-destination pair $w \in W$, flow feasibility is characterized by the conservation and nonnegativity constraints

$$
\begin{aligned}
\sum_{p \in K_{w}} h_{p}(\alpha)=d_{w} \psi_{w}(\alpha) & \forall w \in W, \quad \forall \alpha \\
h_{p}(\alpha) \geq 0 & \forall p \in K_{w}, \quad \forall \alpha .
\end{aligned}
$$

The corresponding arc flows are obtained from the balance equations:

$$
x_{a}(\alpha)=\sum_{w \in W} \sum_{p \in K_{w}} \delta_{a p} h_{p}(\alpha) \quad \forall a \in A, \quad \forall \alpha
$$

where

$$
\delta_{a p}= \begin{cases}1 & \text { if } \operatorname{arc} a \text { belong to path } p \\ 0 & \text { otherwise }\end{cases}
$$

are the coefficients of the arc - path incidence matrix $\Delta$, i.e., in matrix form: $x(\alpha)=\Delta h(\alpha)$. Based on this notation the sets of path (respectively arc) flow density vectors take the form

$$
\begin{aligned}
& H(\alpha)=\left\{h(\alpha) \geq 0: \sum_{p \in K_{w}} h_{p}(\alpha)=d_{w} \psi_{w}(\alpha), w \in W\right\} \\
& X(\alpha)=\Delta H(\alpha)=\{x(\alpha): \exists h(\alpha) \in H(\alpha): x(\alpha)=\Delta h(\alpha)\} .
\end{aligned}
$$

For each value of the parameter $\alpha$, the flow density $x(\alpha)$ solves the mathematical program (see Marcotte 1999)

$$
\min _{x(\alpha) \in X(\alpha)} \int_{0}^{\infty} \sum_{a \in A}\left(T_{a}+\alpha C_{a}\right) x_{a}(\alpha) d \alpha .
$$

By regrouping the fares and delays into respective vectors $T$ and $C$, we obtain the infinitedimensional optimization problem

$$
\min _{x \in X}\langle T+\alpha C, x\rangle
$$

where $X$ is the set of square-integrable vector functions defined as

$$
X=\left\{x \in\left\{L^{2}\left[0, \alpha_{\max }\right)\right\}^{|A|}: x(\alpha) \in X(\alpha), \forall \alpha\right\}
$$

where $\langle\cdot, \cdot\rangle$ denotes the scalar product in $\left\{L^{2}\left[0, \alpha_{\max }\right)\right\}^{|A|}$ :

$$
\langle u, v\rangle=\int_{0}^{\alpha_{\max }}\langle u(\alpha), v(\alpha)\rangle d \alpha
$$

This demand assignment problem with product differentiation corresponds to a parametric infinite dimensional linear program of the bottleneck type (see Anderson and Nash 1987). It 
will be analyzed in Section 3. At the upper level, the leader determines its profit-maximizing strategic policy by solving the mathematical program

$$
\max _{T_{1}}\left\langle T_{1}, x_{1}\left(T_{1}\right)\right\rangle
$$

where $T_{1}=\left(T_{a}\right)_{a \in A_{1}}$ and $x_{1}\left(T_{1}\right)$ is the demand for the products or services offered by the leader firm. For a given feasible policy $T_{1}, x_{1}\left(T_{1}\right)$ is the partial solution, with respect to the arcs controlled by the leader, of the demand assignment problem (5). We can now formulate the strategic policy design problem as the bilevel program

$$
\begin{array}{lll}
\max _{T_{1} \geq 0} & \left\langle T_{1}, x_{1}\right\rangle & \\
\min _{x, h} & \int_{0}^{\alpha_{\max }} \sum_{a \in A}\left(T_{a}+\alpha C_{a}\right) x_{a}(\alpha) d \alpha & \\
& x_{a}(\alpha)=\sum_{w \in W} \sum_{p \in K_{w}} \delta_{a k} h_{p}(\alpha), & \forall a \in A, \quad \forall \alpha \\
& \sum_{p \in K_{w}} h_{p}(\alpha)=d_{w} \psi_{w}(\alpha), & \forall w \in W, \quad \forall \alpha \\
& h_{p}(\alpha) \geq 0 & \forall p \in K_{w}, \quad \forall w \in W, \quad \forall \alpha
\end{array}
$$

In this vertical format, it is understood that $x_{1}$ is the partial solution of the lower level minimization problem, parameterized by the price vector $T_{1}$.

\section{The demand (lower level) assignment problem}

In this section, we analyze the structure of the demand assignment problem. To this end, we express the incidence matrix $\Delta$ as

$$
\Delta=\left(\begin{array}{cccccc}
B_{1}^{1} & B_{1}^{2} & \ldots & B_{1}^{w} & \ldots & B_{1}^{|W|} \\
B_{2}^{1} & B_{2}^{2} & \ldots & B_{2}^{w} & \ldots & B_{2}^{|W|}
\end{array}\right),
$$

where submatrices $B_{i}^{w}$ are of dimension $\left|A_{i}\right| \times\left|K_{w}\right|$. Based on this notation, the generalized path cost vector is given by the affine function

$$
\Delta^{t}(T+\alpha C)=D\left(T_{1}\right)+\alpha G
$$

with $D\left(T_{1}\right)=\Delta^{t} T$ and $G=\Delta^{t} C$. Using this notation, the demand assignment problem that determines the market shares of the services (paths) reduces to

$$
\min _{h \in H}\left\langle D\left(T_{1}\right)+\alpha G, h\right\rangle
$$


Restricting our attention to a single path $p \in K_{w}$ we express $D\left(T_{1}\right)$ as the affine function

$$
D_{p}\left(T_{1}\right)=\left\langle b_{1}^{w p}, T_{1}\right\rangle+\left\langle b_{2}^{w p}, T_{2}\right\rangle
$$

where $b_{i}^{p w}$ is the $p$ th row of the transposed matrix $\left(B_{i}^{w}\right)^{t}$. From now on we will, for notational simplicity, only consider a single origin and, without loss of generality, assume that the demand is equal to one.

We now introduce an order relation among the paths that will be essential in characterizing market shares.

Definition 3.1 A path $p$ is dominated at $T_{1}$ if, for all nonnegative values of $\alpha$ :

$$
D_{p}\left(T_{1}\right)+\alpha G_{p}>\min _{q \in K}\left\{D_{q}\left(T_{1}\right)+\alpha G_{q}\right\} .
$$

Conversely, if there exists a value $\alpha$ for which

$$
D_{p}\left(T_{1}\right)+\alpha G_{p} \leq \min _{q \in K}\left\{D_{q}\left(T_{1}\right)+\alpha G_{q}\right\}
$$

we say that the path $p$ is undominated.

If a path is dominated at $T_{1}$, clearly it is dominated in a neighborhood around $T_{1}$.

Definition 3.2 A path $p$ is called weakly undominated at $T_{1}$ if there exists a unique value of $\alpha$ such that

$$
D_{p}\left(T_{1}\right)+\alpha G_{p} \leq \min _{q \in K}\left\{D_{q}\left(T_{1}\right)+\alpha G_{q}\right\},
$$

A path $p$ which is undominated but not weakly undominated is strongly undominated.

We will see that, if a path is strongly undominated at $T_{1}$, then there exist breakpoints $\left(\alpha_{p}\left(T_{1}\right)\right.$ and $\left.\alpha_{p+1}\left(T_{1}\right)\right)$ such that the dominance relationship holds strongly over the open interval $\left(\alpha_{p}\left(T_{1}\right), \alpha_{p+1}\left(T_{1}\right)\right)$, and weakly at the endpoints of the interval.

Throughout the paper, we will make the following mild assumption, which rules out degeneracy situations (infinite profit, overlapping path cost functions, etc).

\section{Assumption $\mathbf{H}_{0}$}

(i) $G_{p} \neq G_{q}$ whenever $p \neq q$.

(ii) For any $T_{1} \geq 0$, there exists at least one strongly undominated path. 
(iii) There exists a value $\alpha_{\max }$ such that $\psi(\alpha)=0$ whenever $\alpha \geq \alpha_{\max }$.

The dominance relationship allows us to characterize the market structure at a price vector $T_{1}$, independently of the actual market shares, in the following way:

Definition 3.3 Associated with a price vector $T_{1}$, we associate the market structure $(\mathcal{S U}, \mathcal{W U}, \mathcal{D})$ as the triplet composed of the sets of strongly undominated, weakly undominated and dominated paths, respectively.

Let $M$ denote the number of strongly undominated paths. We set $\mathcal{S U}=\left\{p_{i_{k}}\right\}_{k \in[1 . . M]} \subseteq$ $K, \mathcal{W U}=\left\{p_{j_{k}}\right\}$ and $\mathcal{D}=\left\{p_{l_{k}}\right\}$ and, without loss of generality, assume that the scalars $G_{p}$ are sorted in decreasing order, i.e.,

$$
G_{i_{1}}>G_{i_{2}}>\ldots>G_{i_{M}}
$$

Note that, in all rigour, one should have written $G_{p_{i_{k}}}$ in the above. Next, we introduce

$$
\begin{aligned}
& \alpha_{i_{k}}=-\frac{D_{i_{k+1}}\left(T_{1}\right)-D_{i_{k}}\left(T_{1}\right)}{G_{i_{k+1}}-G_{i_{k}}}, \quad k=1, \ldots, M-1, \\
& \alpha_{i_{0}}=0 \\
& \alpha_{i_{M}}=\alpha_{\max } .
\end{aligned}
$$

One possible configuration is illustrated in Figure 2 where, for a given value of the fare vector $T_{1}$, paths 1,2 and 3 are strongly undominated, path 5 is dominated and path 4 is weakly undominated. The market shares of each path are obtained by integrating the density function $\psi$ over the interval where this path achieves the lowest generalized cost.

The next theorem provides an analytic characterization of the market structure, for a given price vector $T$.

Theorem 3.1 Under Assumption $H_{0}$, the system has the market structure $(\mathcal{S U}, \mathcal{W U}, \mathcal{D})$ at $T_{1}$ if and only if the following conditions hold

(i) $0=\alpha_{i_{0}}<\alpha_{i_{1}}<\alpha_{i_{2}}<\ldots<\alpha_{i_{M-1}}<\alpha_{i_{M}}=\alpha_{\max }$.

(ii) If $p_{j} \in \mathcal{D}$, then either 


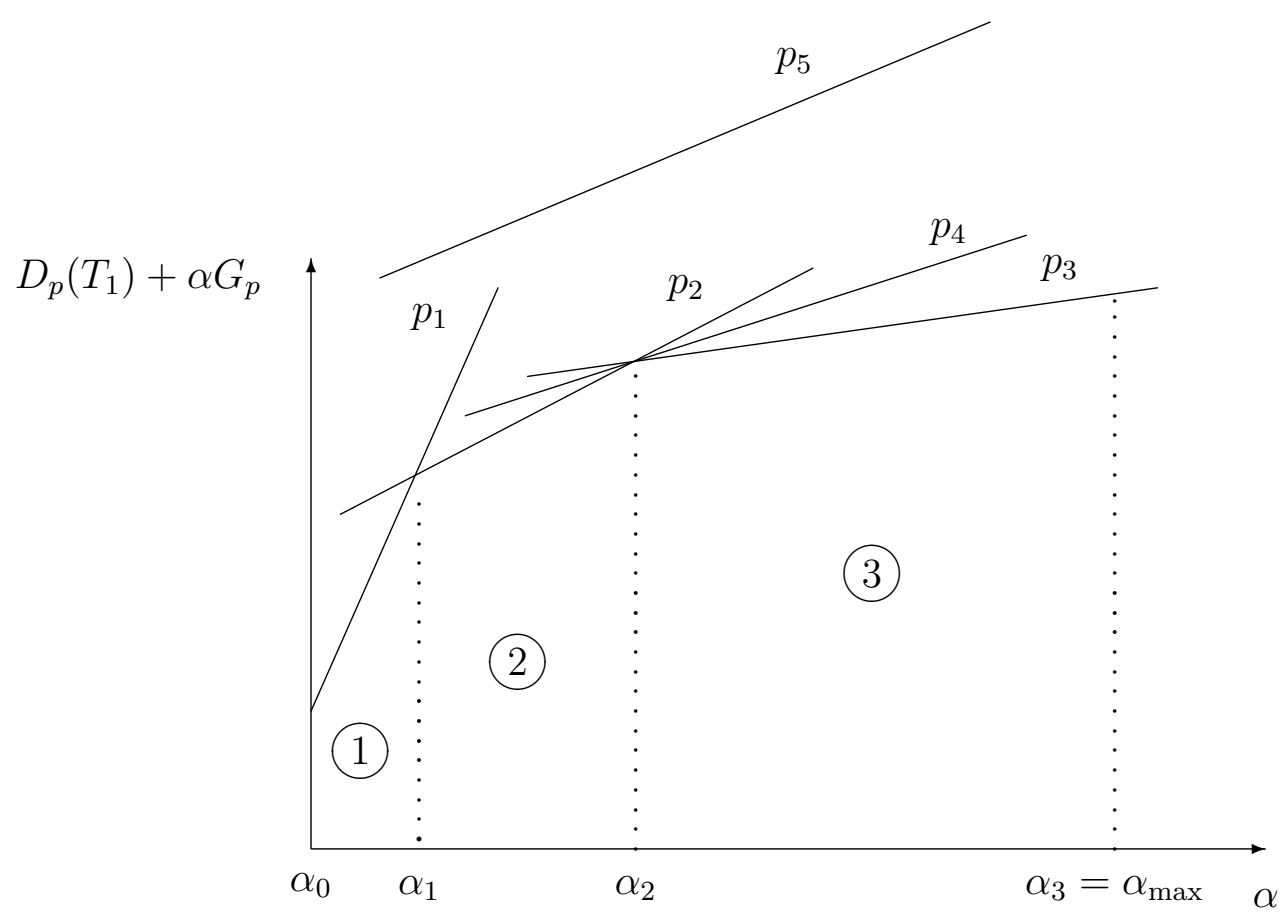

Figure 2: Market structure

- inequality $G_{i_{k}}>G_{j}>G_{i_{k+1}}$ holds for some index $k \in[1 . . M-1]$, with

$$
\beta_{j_{k}}>\alpha_{i_{k}}>\beta_{j_{k+1}}
$$

where $\beta_{j_{k}}=-\frac{D_{j}\left(T_{1}\right)-D_{i_{k}}\left(T_{1}\right)}{G_{j}-G_{i_{k}}}$ and $\beta_{j_{k+1}}=-\frac{D_{j}\left(T_{1}\right)-D_{i_{k+1}}\left(T_{1}\right)}{G_{j}-G_{i_{k+1}}}$;

- inequality $G_{i_{M}}>G_{j}$ holds, and

$$
\beta_{j}=-\frac{D_{j}\left(T_{1}\right)-D_{i_{M}}\left(T_{1}\right)}{G_{j}-G_{i_{M}}}>\alpha_{i_{M}}
$$

- inequality $G_{j}>G_{i_{1}}$ holds, and

$$
\beta_{j}=-\frac{D_{j}\left(T_{1}\right)-D_{i_{1}}\left(T_{1}\right)}{G_{j}-G_{i_{1}}}<0 .
$$

(iii) If $p_{j} \in \mathcal{W U}$, then equality holds for either (15) or (16).

\section{Proof.}

- NECESSITY 
(i) From the labelling of the strongly undominated paths, one has:

$$
D_{i_{1}}\left(T_{1}\right)<D_{i_{2}}\left(T_{1}\right)<\ldots<D_{i_{M}}\left(T_{1}\right)
$$

If $\alpha_{i_{k-1}}>\alpha_{i_{k}}$ for some $k \in[1 . . M-1]$ then

$$
-\frac{D_{i_{k+1}}\left(T_{1}\right)-D_{i_{k}}\left(T_{1}\right)}{G_{i_{k+1}}-G_{i_{k}}}<-\frac{D_{i_{k}}\left(T_{1}\right)-D_{i_{k-1}}\left(T_{1}\right)}{G_{i_{k}}-G_{i_{k-1}}} .
$$

Let

$$
\hat{\alpha}=-\frac{D_{i_{k+1}}\left(T_{1}\right)-D_{i_{k-1}}\left(T_{1}\right)}{G_{i_{k+1}}-G_{i_{k-1}}}
$$

By construction, $\hat{\alpha} \in\left[\alpha_{i_{k}}, \alpha_{i_{k-1}}\right]$. It follows that

$$
\begin{array}{ll}
D_{i_{k}}\left(T_{1}\right)+\alpha G_{i_{k}}>D_{i_{k-1}}\left(T_{1}\right)+\alpha G_{i_{k-1}} & \text { for } \quad \alpha \leq \hat{\alpha} \\
D_{i_{k}}\left(T_{1}\right)+\alpha G_{i_{k}}>D_{i_{k+1}}\left(T_{1}\right)+\alpha G_{i_{k+1}} & \text { for } \quad \alpha>\hat{\alpha}
\end{array}
$$

which implies that path $p_{i_{k}}$ is dominated, a contradiction.

If $\alpha_{i_{k-1}}=\alpha_{i_{k}}$ holds, then

$$
D_{i_{k}}\left(T_{1}\right)+\alpha G_{i_{k}}>D_{i_{k-1}}\left(T_{1}\right)+\alpha G_{i_{k-1}}
$$

for $\alpha<\alpha_{i_{k-1}}=\alpha_{i_{k}}$.

$$
D_{i_{k}}\left(T_{1}\right)+\alpha G_{i_{k}}>D_{i_{k+1}}\left(T_{1}\right)+\alpha G_{i_{k+1}}
$$

for $\alpha>\alpha_{i_{k}}$, which implies that path $p_{i_{k}}$ is weakly undominated, a contradiction.

\section{(ii) Case 1}

If $\beta_{j_{k}} \leq \alpha_{i_{k}}$, i.e.,

$$
\frac{D_{i_{k}}\left(T_{1}\right)-D_{j}\left(T_{1}\right)}{G_{j}-G_{i_{k}}} \leq \alpha_{i_{k}}
$$

then we have

$$
D_{i_{k}}\left(T_{1}\right)-D_{j}\left(T_{1}\right) \geq\left(G_{j}-G_{i_{k}}\right) \alpha_{i_{k}}
$$

or, equivalently,

$$
D_{i_{k}}\left(T_{1}\right)+\alpha_{i_{k}} G_{i_{k}} \geq D_{j}\left(T_{1}\right)+\alpha_{i_{k}} G_{j}
$$

which implies that $p_{j}$ is undominated, a contradiction. The reverse inequality (15) follows from a similar argument. 


\section{Case 2}

In this case, we have that $G_{i_{M}}>G_{j}$. If

$$
\beta_{j}=-\frac{D_{j}\left(T_{1}\right)-D_{i_{M}}\left(T_{1}\right)}{G_{j}-G_{i_{M}}} \leq \alpha_{i_{M}}
$$

then $D_{i_{M}}\left(T_{1}\right)-D_{j}\left(T_{1}\right) \geq \alpha_{i_{M}}\left(G_{j}-G_{i_{M}}\right)$, i.e., $D_{i_{M}}\left(T_{1}\right)+\alpha_{i_{M}} G_{i_{M}} \geq D_{j}\left(T_{1}\right)+\alpha_{i_{M}} G_{j}$, a contradiction.

\section{Case 3}

The proof is similar to that of (ii) (Case 2).

(iii) Using the results of (i) and (ii), the proof of (iii) is readily obtained.

\section{- SUFFICIENCY}

(i) From the assumption, we observe that

$$
D_{i_{M}}\left(T_{1}\right)+\alpha_{i_{k-1}} G_{i_{M}}>\ldots>D_{i_{k+1}}\left(T_{1}\right)+\alpha_{i_{k-1}} G_{i_{k+1}}>D_{i_{k}}\left(T_{1}\right)+\alpha_{i_{k-1}} G_{i_{k}}
$$

and

$$
D_{i_{M}}\left(T_{1}\right)+\alpha_{i_{k}} G_{i_{M}}>\ldots>D_{i_{k+1}}\left(T_{1}\right)+\alpha_{i_{k}} G_{i_{k+1}}=D_{i_{k}}\left(T_{1}\right)+\alpha_{i_{k}} G_{i_{k}}
$$

It follows that

$$
D_{i_{j}}\left(T_{1}\right)+\alpha G_{i_{j}}>D_{i_{k}}\left(T_{1}\right)+\alpha G_{i_{k}}, j \in[k+1 . . M] \quad \text { for } \quad \forall \alpha \in\left(\alpha_{i_{k-1}}, \alpha_{i_{k}}\right) .
$$

On the other hand, we have

$$
\begin{aligned}
D_{i_{1}}\left(T_{1}\right)+\alpha G_{i_{1}} & >D_{i_{2}}\left(T_{1}\right)+\alpha G_{i_{2}} \text { for } \quad \alpha>\alpha_{i_{1}} \\
D_{i_{2}}\left(T_{1}\right)+\alpha G_{i_{2}} & >D_{i_{3}}\left(T_{1}\right)+\alpha G_{i_{3}} \text { for } \quad \alpha>\alpha_{i_{2}} \\
& \vdots \\
D_{i_{k-1}}\left(T_{1}\right)+\alpha G_{i_{k-1}} & >D_{i_{k}}\left(T_{1}\right)+\alpha G_{i_{k}} \text { for } \quad \alpha>\alpha_{i_{k-1}} .
\end{aligned}
$$

The above inequalities imply that $p_{i_{k}}$ is strongly undominated.

(ii) 


\section{Case 1}

If $\beta_{j_{k}}>\alpha_{i_{k}}>\beta_{j_{k+1}}$, then

$$
\begin{aligned}
& D_{j}\left(T_{1}\right)+\alpha G_{j}>D_{i_{k}}\left(T_{1}\right)+\alpha G_{i_{k}} \quad \text { for } \quad \alpha<\alpha_{i_{k}}<\beta_{j_{k}} \\
& D_{j}\left(T_{1}\right)+\alpha G_{j}>D_{i_{k+1}}\left(T_{1}\right)+\alpha G_{i_{k+1}} \text { for } \quad \alpha>\alpha_{i_{k}}>\beta_{j_{k+1}} \text {. }
\end{aligned}
$$

This implies that $p_{j}$ is dominated.

\section{Case 2}

If $\beta_{j}>\alpha_{i_{M}}$, i.e., $\left(D_{j}\left(T_{1}\right)-D_{i_{M}}\left(T_{1}\right)\right) /\left(G_{i_{M}}-G_{j}\right)>\alpha_{i_{M}}$, we have

$$
D_{j}\left(T_{1}\right)+\alpha G_{j}>D_{i_{M}}\left(T_{1}\right)+\alpha G_{i_{M}} \text { for all } \quad \alpha \leq \alpha_{i_{M}}
$$

and path $p_{j}$ is dominated.

\section{Case 3}

The proof is similar to that of Case 2 .

(iii) Obvious. This completes the proof.

The analysis of the market structure allows to perform sensitivity analysis of the lower level problem with respect to the price vector $T_{1}$. First, we investigate the regions where the market structure is invariant:

$$
\Lambda_{\mathcal{S W D}}=\left\{T_{1} \geq 0: p_{i_{k}} \in \mathcal{S U}, k \in[1 . . M] ; p_{j_{k}} \in \mathcal{W U}, k \in[1 . . N] ; p_{l_{k}} \in \mathcal{D}, k \in[1 . . S]\right\}
$$

It follows from Theorem 3.1 that the set $\Lambda_{\mathcal{S W D}}$ is a polyhedron where all demand is assigned to a fixed set of dominated paths that share the total market. Customers are associated with segments $\left[\alpha_{i-1}, \alpha_{i}\right]$ and the market share of a strongly undominated path $p_{i}$ is obtained by integrating the density function $\psi$ over the corresponding interval. Within an interval, the generalized cost of customers are all minimized by the same strongly undominated path $p_{i}$.

Geometrically, the whole strategic policy space $R^{\left|A_{1}\right|}$ can be partitioned into regions, each region having its own structure, i.e.,

$$
R^{\left|A_{1}\right|}=\bigcup \Lambda_{\mathcal{S W D}}
$$




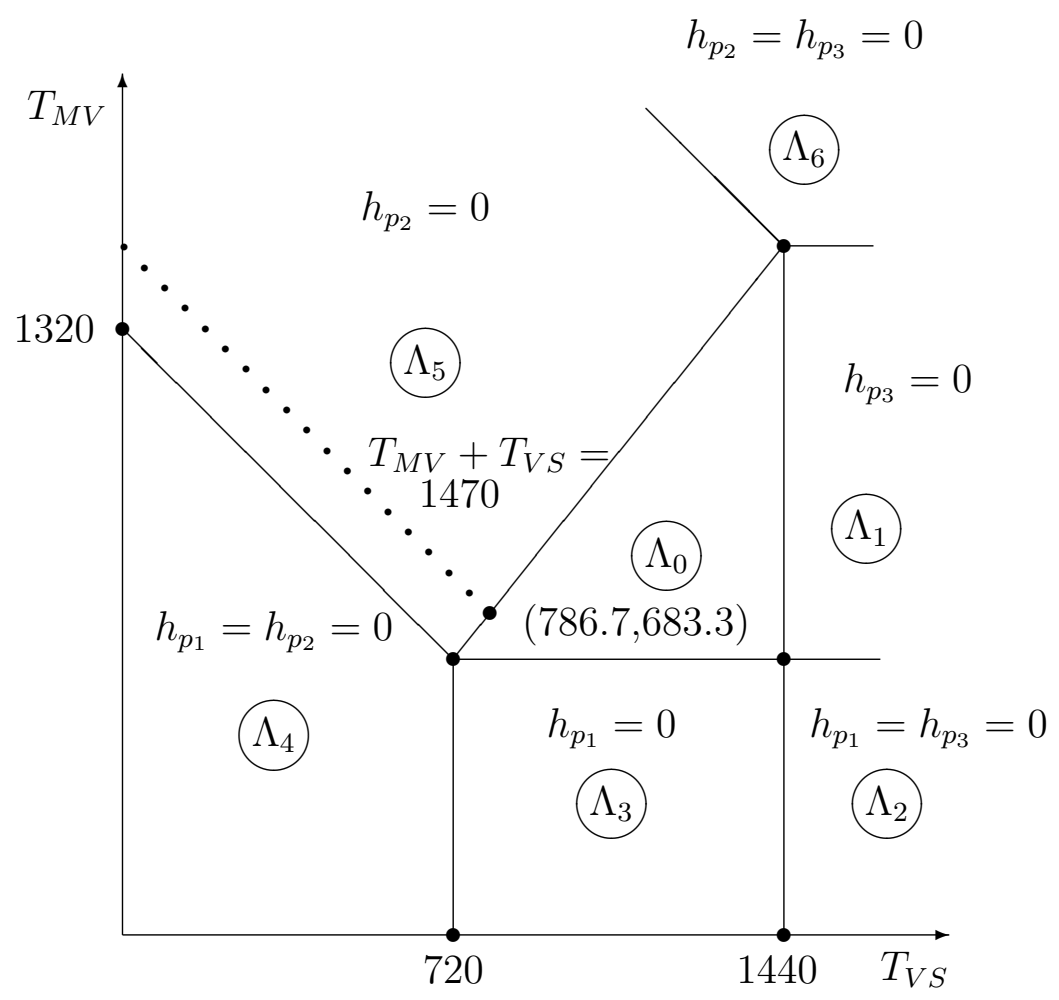

Figure 3: Market structure for the Montreal - Shanghai example

Figure 3 illustrates the partition corresponding to the Montreal - Shanghai market presented in the introduction. Each region $\Lambda_{i}$ is characterized by its set of undominated paths or, equivalently, by the set of paths with null flows. In the interior of the regions, all undominated paths are strongly undominated. For instance, all paths are undominated in region $\Lambda_{0}$ while, in the interior of $\Lambda_{6}$, only path $p_{1}$ is strongly undominated. In this example, the density function $\psi$ is uniform over the interval $\left[0, \alpha_{\max }\right]$ and any point on the dotted line is an optimal solution of the pricing problem.

\section{Sensitivity analysis of demand side}

In this section, we study the sensitivity of the lower level solution to the price vector $T_{1}$. To this end, we need to solve, for each value of $\alpha$, the parameterized problem

$$
\operatorname{LP}\left(T_{1}, \alpha\right): \quad \min _{h(\alpha) \in H(\alpha)}\left\langle D\left(T_{1}\right)+\alpha G, h(\alpha)\right\rangle .
$$

Let $M\left(T_{1}\right)$ denote the number of strongly undominated paths at $T_{1}$. If the density function $\psi$ is strictly positive over the interval $\left[0, \alpha_{\max }\right.$ ), the (almost everywhere) unique solution of $\operatorname{LP}\left(T_{1}, \alpha\right)$ is $h(\alpha)=Y^{i(\alpha)} \psi(\alpha)$, where $Y^{i(\alpha)}$ is the extremal point of the simplex: $\{y$ : 
$\left.\sum_{i \in\left[1 . . M\left(T_{1}\right)\right]} y_{i}=1, y_{i} \geq 0\right\}$ associated with $i(\alpha)$ that satisfies

$$
D_{i(\alpha)}\left(T_{1}\right)+\alpha G_{i(\alpha)}=\min _{j \in\left[1 . . M\left(T_{1}\right)\right]} D_{j}\left(T_{1}\right)+\alpha G_{j}
$$

Note that this point is unique except at the critical points $\alpha_{i}$ who are in finite number.

Theorem 4.1 For fixed $T_{1} \in \Lambda_{\mathcal{S W D}}$, the solution of the infinite-dimensional lower level program is unique (almost everywhere) and given by:

$$
h(\alpha)=Y^{i_{k}} \psi(\alpha), \text { for } \alpha \in\left[\alpha_{i_{k-1}}, \alpha_{i_{k}}\right), \quad k \in[1 . . M]
$$

and the total path flow vector $h\left(T_{1}\right)$ is unambiguously defined.

Proof. The value function $\phi(\alpha)=D_{i(\alpha)}\left(T_{1}\right)+\alpha G_{i(\alpha)}$ is the piecewise affine concave function of its scalar argument $\alpha$ which interpolates the points

$$
(0, \phi(0)),\left(\alpha_{i_{1}}, \phi\left(\alpha_{i_{1}}\right)\right), \ldots,\left(\alpha_{i_{M-1}}, \phi\left(\alpha_{i_{M-1}}\right)\right) .
$$

On each subinterval $\left(\alpha_{i_{k-1}}, \alpha_{i_{k}}\right)$ its slope is equal to $G_{i_{k}}$. In the interior of each subinterval, the optimum of $\operatorname{LP}\left(T_{1}, \alpha\right)$ is achieved at an extreme point $Y^{i_{k}}$ of the simplex. At a breakpoint $\alpha_{i_{k}}$, the optimum of $\operatorname{LP}\left(T_{1}, \alpha\right)$ is achieved at either one of the extreme points $Y^{j}$, say $j \in J_{k}$, where $J_{k}$ denotes the index set of extreme points; in that case $h\left(\alpha_{i_{k}}\right)$ can be arbitrarily set to $\psi\left(\alpha_{i_{k}}\right)$ times a convex combination of the $Y^{j}$ 's, for $j$ in $J_{k}$. Since the number of breakpoints is finite, the total path flow $h\left(T_{1}\right)$ is unique.

Under Assumption $H_{0}$ and the market structure $(\mathcal{S U}, \mathcal{W U}, \mathcal{D})$ at $T_{1}$ exactly one of the following three inequalities must hold for a given undominated path $p_{j}$ :

1. $G_{i_{u}}>G_{j}>G_{i_{l}}$, where $l=\arg \min \left\{k \mid G_{i_{k}}<G_{j}, p_{i_{k}} \in \mathcal{S U}\right\}$ and $u=\arg \max \left\{k \mid G_{i_{k}}>G_{j}, p_{i_{k}} \in \mathcal{S U}\right\}$;

2. $G_{i_{M}}>G_{j}$ for $p_{i_{M}} \in \mathcal{S U}$;

3. $G_{j}>G_{i_{1}}$ for $p_{i_{1}} \in \mathcal{S U}$.

We will refer to paths $p_{i_{u}}$ and $p_{i_{l}}$ (or $p_{i_{M}}, p_{i_{1}}$ ) as the $\mathcal{S U}$ (respectively left and right) neighbors of $p_{j}$.

The characterization of $\mathcal{W U}$ neighbors is a little more involved. Let

$$
L_{u}=\left\{k \in \mathcal{W U} \mid G_{i_{u}}>G_{k}>G_{j}\right\} .
$$


If $L_{u}$ is nonempty, we define the left $\mathcal{W U}$ neighbor of path $p_{j}$ as the path $p_{j_{u}}$ such that

$$
G_{j_{u}}=\min \left\{G_{k} \mid k \in L_{u}\right\}
$$

Symmetrically, a right $\mathcal{W U}$ neighbor of path $p_{j}$ satisfies

$$
G_{j_{l}}=\max \left\{G_{k} \mid k \in L_{l}\right\}
$$

whenever the set $L_{l}=\left\{k \in \mathcal{W U} \mid G_{j}>G_{k}>G_{i_{l}}\right\}$ is nonempty.

The notion of neighbor is key in deriving results about the local behaviour of the revenue function. For simplicity, we analyze its behaviour in the case where the set of $\mathcal{W U}$ neighbors is empty, and then proceed with some remarks about the degenerate cases. In the course of the proof, one may obviously discard dominated paths, with the exception of the path $p_{j}$ under consideration.

Theorem 4.2 Assume that the market structure $(\mathcal{S U}, \mathcal{W U}, \mathcal{D})$ holds at $T_{1}$ and let all paths be strongly undominated, with the possible exception of path $p_{j}$. Then, under Assumption $H_{0}$, we have:

(i) If path $p_{j}$ is strongly undominated and $a \in A_{1}$, then $h_{p_{j}}\left(T_{1}\right)$ is partially differentiable with respect to $T_{a}$.

(i-1) If $a \in p_{j}$, then $h_{p_{j}}$ has a nonpositive partial derivative with respect to $T_{a}$; moreover, if arc a belongs to all $\mathcal{S U}$ neighbors of $p_{j}$, then $\frac{\partial h_{p_{j}}\left(T_{1}\right)}{\partial T_{a}}=0$; otherwise $\frac{\partial h_{p_{j}}\left(T_{1}\right)}{\partial T_{a}}<0$.

(i-2) If a $\notin p_{j}$, then $h_{p_{j}}$ has a nonnegative partial derivative with respect to $T_{a}$; moreover if arc a does not belong to all $\mathcal{S U}$ neighbors of $p_{j}$, then $\frac{\partial h_{p_{j}}\left(T_{1}\right)}{\partial T_{a}}=0$; otherwise $\frac{\partial h_{p_{j}}\left(T_{1}\right)}{\partial T_{a}}>0$.

(ii) If path $p_{j}$ is weakly undominated and $a \in A_{1}$, then $h_{p_{j}}\left(T_{1}\right)$ is partially side differentiable with respect to $T_{a}$.

(ii-1) If $a \in p_{j}$, then $h_{p_{j}}$ has nonnegative partial side derivatives; moreover if arc a belongs to all $\mathcal{S U}$ neighbors of $p_{j}$, then $\frac{\partial h_{p_{j}}\left(T_{1}\right)}{\partial T_{a}}=0$; otherwise $\frac{\partial^{-} h_{p_{j}}\left(T_{1}\right)}{\partial T_{a}}>0$ and $\frac{\partial^{+} h_{p_{j}}\left(T_{1}\right)}{\partial T_{a}}=0$.

(ii-2) If a $\notin p_{j}$, then $p_{j}$ has nonpositive partial side derivatives; moreover, if arc a does not belong to the $\mathcal{S U}$ neighbors of $p_{j}$, then the partial derivatives of $h_{p_{j}}\left(T_{1}\right)$ are equal to zero; otherwise $\frac{\partial^{+} h_{p_{j}}\left(T_{1}\right)}{\partial T_{a}}<0$ and $\frac{\partial^{-} h_{p_{j}}\left(T_{1}\right)}{\partial T_{a}}=0$. 
(iii) If path $p_{j} \in \mathcal{D}$ and $a \in A_{1}$, then $h_{p_{j}}\left(T_{1}\right)$ is partially differentiable with respect to $T_{a}$, and $\frac{\partial h_{p_{j}}\left(T_{1}\right)}{\partial T_{a}}=0$.

\section{Proof.}

(i) Let $p_{j} \in \mathcal{S U}$ at $T_{1}$ and assume that $j=i_{k}$. The demand for path $p_{j}$ is given by

$$
h_{p_{j}}\left(T_{1}\right)=h_{p_{i_{k}}}\left(T_{1}\right)=\int_{\alpha_{i_{k-1}}\left(T_{1}\right)}^{\alpha_{i_{k}}\left(T_{1}\right)} \psi(\alpha) d \alpha, \quad k \in[1 . . M]
$$

with

$$
\begin{aligned}
& \alpha_{i_{k}}\left(T_{1}\right)=-\frac{D_{i_{u}}\left(T_{1}\right)-D_{i_{k}}\left(T_{1}\right)}{G_{i_{u}}-G_{i_{k}}}, k \in[1 . . M-1] \\
& \alpha_{i_{M}}=\alpha_{\max } ; \\
& \alpha_{i_{k-1}}\left(T_{1}\right)=-\frac{D_{i_{l}}\left(T_{1}\right)-D_{i_{k}}\left(T_{1}\right)}{G_{i_{l}}-G_{i_{k}}}, k \in[1 . . M-1] \\
& \alpha_{i_{0}}=0 .
\end{aligned}
$$

Since $\alpha_{i_{k}}\left(T_{1}\right)$ is a linear function of $T_{1}$ then, for sufficiently small perturbation

$$
\delta T_{1}=\left(0, \ldots, 0,\left(\delta T_{1}\right)_{a}, 0 \ldots, 0\right)^{t}
$$

the market structure (18) holds at $T_{1}+\delta T_{1}$, i.e.,

$$
0=\alpha_{i_{0}}<\alpha_{i_{1}}\left(T_{1}+\delta T_{1}\right)<\alpha_{i_{2}}\left(T_{1}+\delta T_{1}\right)<\ldots<\alpha_{i_{M-1}}\left(T_{1}+\delta T_{1}\right)<\alpha_{i_{M}}=\alpha_{\max }
$$

and the flow on path $p_{j}$ is given by

$$
h_{p_{j}}\left(T_{1}+\delta T_{1}\right)=\int_{\alpha_{i_{k-1}}\left(T_{1}+\delta T_{1}\right)}^{\alpha_{i_{k}}\left(T_{1}+\delta T_{1}\right)} \psi(\alpha) d \alpha, \quad k \in[1 . . M] .
$$

Therefore

$$
\frac{\partial h_{p_{j}}\left(T_{1}\right)}{\partial T_{a}}=\frac{\partial \alpha_{i_{k}}\left(T_{1}\right)}{\partial T_{a}} \psi\left(\alpha_{i_{k}}\left(T_{1}\right)\right)-\frac{\partial \alpha_{i_{k-1}}\left(T_{1}\right)}{\partial T_{a}} \psi\left(\alpha_{i_{k-1}}\left(T_{1}\right)\right)
$$

which settles cases (i-1) and (i-2).

(ii) Let $p_{j}$ be weakly undominated. This implies that $h_{p_{j}}\left(T_{1}\right)=0$. We will only give a proof for the case where the inequality $G_{i_{u}}>G_{j}>G_{i_{l}}$ holds for some $k \in[1 . . M-1]$, since a similar argument is valid for the cases where $G_{i_{M}}>G_{j}$ or $G_{j}>G_{i_{1}}$. 
- Case (ii-1)

Let $a \in p_{j}$. If arc $a$ belongs to all SU neighbours of $p_{j}$, then $h_{p_{j}}\left(T_{1}+\delta T_{1}\right)=0$.

Otherwise

$$
h_{p_{j}}\left(T_{1}+\delta T_{1}\right)=\int_{\beta_{j_{k}}\left(T_{1}+\delta T_{1}\right)}^{\beta_{j_{k+1}}\left(T_{1}+\delta T_{1}\right)} \psi(\alpha) d \alpha
$$

where

$$
\beta_{j_{k}}\left(T_{1}+\delta T_{1}\right)=-\frac{D_{j}\left(T_{1}+\delta T_{1}\right)-D_{i_{l}}\left(T_{1}+\delta T_{1}\right)}{G_{j}-G_{i_{l}}}
$$

and

$$
\beta_{j_{k+1}}\left(T_{1}+\delta T_{1}\right)=-\frac{D_{j}\left(T_{1}+\delta T_{1}\right)-D_{i_{u}}\left(T_{1}+\delta T_{1}\right)}{G_{j}-G_{i_{u}}} .
$$

From (29)-(31), the desired results follows.

- Case (ii-2).

Let $a \notin p_{j}$. If $\left(\delta T_{1}\right)_{a} \leq 0$ or $\left(\delta T_{1}\right)_{a}>0$ and $a$ is neither in $p_{i_{k+1}}$ nor in $p_{i_{k}}$, then $h_{p_{j}}\left(T_{1}+\delta T_{1}\right)=0$. Otherwise (29)-(31) is still valid and the results holds.

(iii) If $p_{j}$ is dominated, then $h_{p_{j}}\left(T_{1}\right)=h_{p_{j}}\left(T_{1}+\delta T_{1}\right)=0$ for any sufficiently small $\left(\delta T_{1}\right)_{a}$, i.e., $\frac{\partial h_{p_{j}}\left(T_{1}\right)}{\partial T_{a}}=0$ for all $a \in A_{1}$.

Corollary 4.1 Under the assumptions of Theorem 4.2, the path flow functions $h_{p_{j}}$ are locally Lipschitz continuous with respect to $T_{1}$.

Proof. In the proof of Theorem 4.2, we showed that every breakpoint $\alpha_{i_{k}}\left(T_{1}\right)$ and $\beta_{j_{k}}(T)$ is linear in $T_{1}$ and that the partial side derivatives are bounded. It follows that the path flow $h_{j}\left(T_{1}\right), j \in K$ is Lipschitz continuous on some neighborhood of $T_{1}$.

In the last part of this section, we consider the case where $p_{j} \in \mathcal{S U}$ and the inequality $G_{i_{l}}>G_{j_{l}}>G_{j}>G_{j_{u}}>G_{i_{u}}$ holds. For simplicity, only one pair of $\mathcal{W U}$ is considered. Note that the demand of $p_{j}$ at $T_{1}$ is given by (29). Following a small perturbation $\delta T_{1}$, the market structure (18) at $T_{1}+\delta T_{1}$ is modified, and the flow on path $p_{j}$ becomes

$$
h_{p_{j}}\left(T_{1}+\delta T_{1}\right)=\int_{\alpha_{l}\left(T_{1}+\delta T_{a}\right)}^{\alpha_{u}\left(T_{1}+\delta T_{a}\right)} \psi(\alpha) d \alpha
$$

where the values of the new breakpoints $\alpha_{u}\left(T_{1}+\delta T_{a}\right)$ and $\alpha_{l}\left(T_{1}+\delta T_{a}\right)$ are given in Table 2 for several cases. In the first three columns, a ' 1 ' indicates that arc $a$ belongs to the relevant 


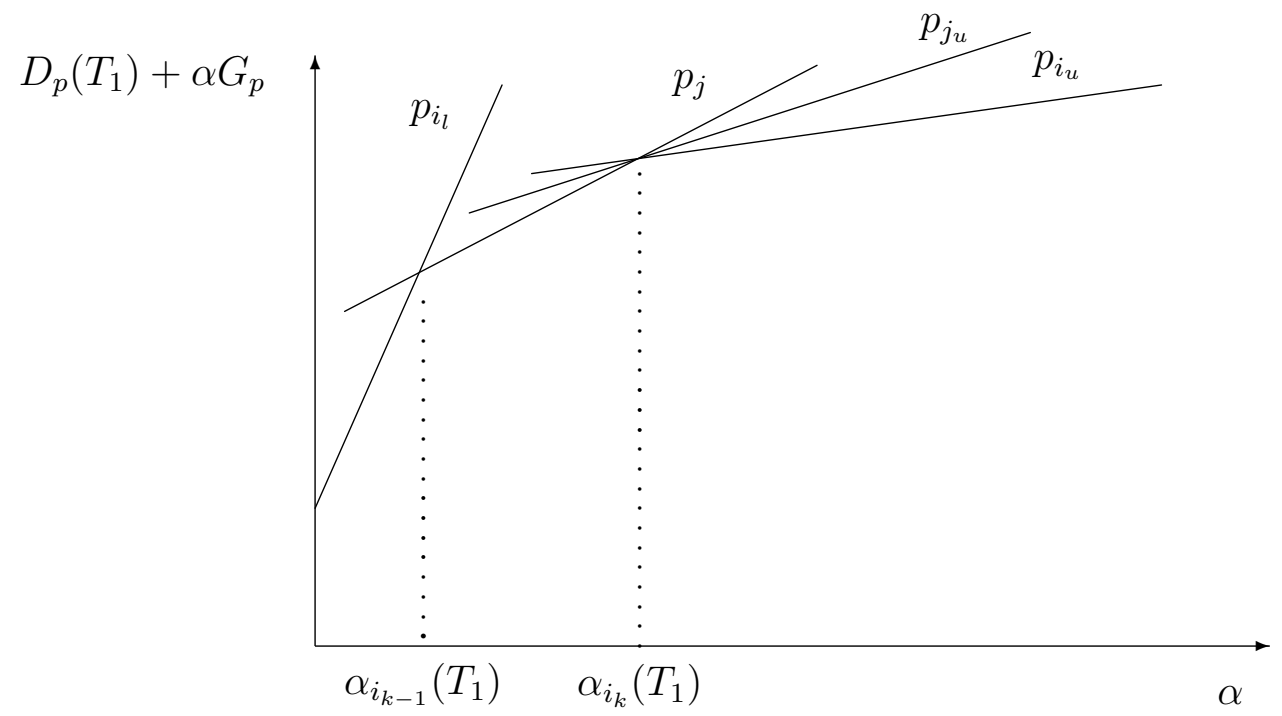

Figure 4: Sensitivity of the market structure

path. From Figure 4, it is clear that the market structure is modified if only $a \in p_{j}$. Once sensitivity of the breakpoints is obtained, the partial (side) derivative of the path flow $h_{p_{j}}$ with respect to $T_{a}$ readily follows:

$$
\frac{\partial h_{p_{j}}\left(T_{1}\right)}{\partial T_{a}}=\frac{\partial \alpha_{u}\left(T_{1}\right)}{\partial T_{a}} \psi\left(\alpha_{u}\left(T_{1}\right)\right)-\frac{\partial \alpha_{l}\left(T_{1}\right)}{\partial T_{a}} \psi\left(\alpha_{l}\left(T_{1}\right)\right) .
$$




\begin{tabular}{|c|c|c|c|c|c|c|c|c|}
\hline case & $a \in p_{j}$ & $\begin{array}{c}a \in p_{i_{l}} \\
{\left[a \in p_{i_{u}}\right]}\end{array}$ & $\begin{array}{c}a \in p_{j_{l}} \\
{\left[a \in p_{j_{u}}\right]}\end{array}$ & $\delta T_{a}$ & $\alpha_{l}\left(T_{1}+\delta T_{a}\right)$ & $\frac{\partial \alpha_{l}}{\partial T_{a}}$ & {$\left[\alpha_{u}\left(T_{1}+\delta T_{a}\right)\right]$} & {$\left[\frac{\partial \alpha_{u}}{\partial T_{a}}\right]$} \\
\hline 1 & 1 & 1 & 1 & $+/-$ & $\alpha_{i_{k-1}}\left(T_{1}\right)$ & 0 & $\alpha_{i_{k}}\left(T_{1}\right)$ & 0 \\
\hline 2 & 1 & 1 & 0 & + & $\alpha_{i_{k-1}}\left(T_{1}\right)-\frac{\delta T_{a}}{G_{j}-G_{j_{l}}}$ & $-\frac{1}{G_{j}-G_{j_{l}}}$ & $\alpha_{i_{k}}\left(T_{1}\right)-\frac{\delta T_{a}}{G_{j}-G_{j_{u}}}$ & $-\frac{1}{G_{j}-G_{j_{u}}}$ \\
\hline 3 & 1 & 1 & 0 & - & $\alpha_{i_{k-1}}\left(T_{1}\right)$ & 0 & $\alpha_{i_{k}}\left(T_{1}\right)$ & 0 \\
\hline 4 & 1 & 0 & 1 & + & $\alpha_{i_{k-1}}\left(T_{1}\right)-\frac{\delta T_{a}}{G_{j}-G_{i_{l}}}$ & $-\frac{1}{G_{j}-G_{i_{l}}}$ & $\alpha_{i_{k}}\left(T_{1}\right)-\frac{\delta T_{a}}{G_{i_{u}}-G_{j}}$ & $-\frac{1}{G_{i_{u}}-G_{j}}$ \\
\hline 5 & 1 & 0 & 1 & - & $\alpha_{i_{k-1}}\left(T_{1}\right)-\frac{\delta T_{a}}{G_{j}-G_{j_{l}}}$ & $-\frac{1}{G_{j}-G_{j_{l}}}$ & $\alpha_{i_{k}}\left(T_{1}\right)-\frac{\delta T_{a}}{G_{j}-G_{j_{u}}}$ & $-\frac{1}{G_{j}-G_{j_{u}}}$ \\
\hline 6 & 1 & 0 & 0 & + & $\alpha_{i_{k-1}}\left(T_{1}\right)-\frac{\delta T_{a}}{G_{j}-G_{j_{l}}}$ & $-\frac{1}{G_{j}-G_{j_{l}}}$ & $\alpha_{i_{k}}\left(T_{1}\right)-\frac{\delta T_{a}}{G_{j}-G_{j_{u}}}$ & $-\frac{1}{G_{j}-G_{j_{u}}}$ \\
\hline 7 & 1 & 0 & 0 & - & $\alpha_{i_{k-1}}\left(T_{1}\right)-\frac{\delta T_{a}}{G_{j}-G_{i_{l}}}$ & $-\frac{1}{G_{j}-G_{i_{l}}}$ & $\alpha_{i_{k-1}}\left(T_{1}\right)-\frac{\delta T_{a}}{G_{j_{u}}-G_{j}}$ & $-\frac{1}{G_{j_{u}}-G_{j}}$ \\
\hline 8 & 0 & 0 & 0 & $+/-$ & $\alpha_{i_{k-1}}\left(T_{1}\right)$ & 0 & $\alpha_{i_{k}}\left(T_{1}\right)$ & 0 \\
\hline 9 & 0 & 0 & 1 & + & $\alpha_{i_{k-1}}\left(T_{1}\right)$ & 0 & $\alpha_{i_{k}}\left(T_{1}\right)$ & 0 \\
\hline 10 & 0 & 0 & 1 & - & $\alpha_{i_{k-1}}\left(T_{1}\right)-\frac{\delta T_{a}}{G_{j_{l}}-G_{i_{l}}}$ & $-\frac{1}{G_{j_{l}}-G_{i_{l}}}$ & $\alpha_{i_{k}}\left(T_{1}\right)-\frac{\delta T_{a}}{G_{j}-G_{j_{u}}}$ & $-\frac{1}{G_{j}-G_{j_{u}}}$ \\
\hline 11 & 0 & 1 & 1 & + & $\alpha_{i_{k-1}}\left(T_{1}\right)-\frac{\delta T_{a}}{G_{j}-G_{i_{l}}}$ & $-\frac{1}{G_{j}-G_{i_{l}}}$ & $\alpha_{i_{k}}\left(T_{1}\right)-\frac{\delta T_{a}}{G_{j}-G_{j_{u}}}$ & $-\frac{1}{G_{j_{u}}}$ \\
\hline 12 & 0 & 1 & 1 & - & $\alpha_{i_{k-1}}\left(T_{1}\right)-\frac{\delta T_{a}}{G_{j}-G_{j_{l}}}$ & $-\frac{1}{G_{j}-G_{j_{l}}}$ & $\alpha_{i_{k}}\left(T_{1}\right)-\frac{\delta T_{a}}{G_{j}-G_{i_{u}}}$ & $-\frac{1}{G_{j}-G_{i_{u}}}$ \\
\hline 13 & 0 & 1 & 0 & + & $\alpha_{i_{k-1}}\left(T_{1}\right)$ & 0 & $\alpha_{i_{k}}\left(T_{1}\right)$ & 0 \\
\hline 14 & 0 & 1 & 0 & - & $\alpha_{i_{k-1}}\left(T_{1}\right)-\frac{\delta T_{a}}{G_{j}-G_{i_{l}}}$ & $-\frac{1}{G_{j}-G_{i_{l}}}$ & $\alpha_{i_{k}}\left(T_{1}\right)-\frac{\delta T_{a}}{G_{i_{u}}-G_{j}}$ & $-\frac{1}{G_{i_{u}}-G_{j}}$ \\
\hline
\end{tabular}

Table 2: Price sensitivity of demand function 


\section{A piecewise optimization strategy}

Based on the sensitivity results of Section 4, it is possible to solve the pricing problem by standard descent methods, for a given market structure. If one denotes by $x\left(T_{1}\right)$ and $h\left(T_{1}\right)$ the arc and path flow vectors that correspond to a price schedule $T_{1}$, the leader firm maximizes its revenue by solving the unconstrained mathematical program

$$
\max _{T_{1}} f\left(T_{1}\right)=\left\langle T_{1}, x_{1}\left(T_{1}\right)\right\rangle=\left\langle B_{1} T_{1}, h\left(T_{1}\right)\right\rangle
$$

Restricted to a given area $\Lambda_{i}$ of the market structure $(\mathcal{S U}, \mathcal{W U}, \mathcal{D})$, the objective function takes the form

$$
\begin{aligned}
f_{\Lambda_{i}}\left(T_{1}\right)= & \left\langle B_{1} T_{1}, h\left(T_{1}\right)\right\rangle \\
= & \sum_{k=1}^{M}\left\langle b_{1}^{i_{k}}, T_{1}\right\rangle h_{i_{k}}\left(T_{1}\right) \\
= & \left\langle b_{1}^{i_{1}}, T_{1}\right\rangle \int_{0}^{\alpha_{i_{1}}\left(T_{1}\right)} \psi(\alpha) d \alpha+\left\langle b_{1}^{i_{2}}, T_{1}\right\rangle \int_{\alpha_{i_{1}}\left(T_{1}\right)}^{\alpha_{i_{2}}\left(T_{1}\right)} \psi(\alpha) d \alpha \\
& +\ldots+\left\langle b_{1}^{i_{M}}, T_{1}\right\rangle \int_{\alpha_{i_{M-1}}\left(T_{1}\right)}^{\alpha_{i_{M}}\left(T_{1}\right)} \psi(\alpha) d \alpha \\
= & \left\langle b_{1}^{i_{1}}-b_{1}^{i_{2}}, T_{1}\right\rangle \int_{0}^{\alpha_{i_{1}}\left(T_{1}\right)} \psi(\alpha) d \alpha+\left\langle b_{1}^{i_{2}}-b_{1}^{i_{3}}, T_{1}\right\rangle \int_{0}^{\alpha_{i_{2}}\left(T_{1}\right)} \psi(\alpha) d \alpha \\
& +\ldots+\left\langle b_{1}^{i_{M-1}}-b_{1}^{i_{M}}, T_{1}\right\rangle \int_{0}^{\alpha_{i_{M-1}}\left(T_{1}\right)} \psi(\alpha) d \alpha+\left\langle b_{1}^{i_{M}}, T_{1}\right\rangle .
\end{aligned}
$$

If one sets

$$
g_{j}\left(T_{1}\right)=\left\langle b_{1}^{i_{j-1}}-b_{1}^{i_{j}}, T_{1}\right\rangle \int_{0}^{\alpha_{i_{j-1}}\left(T_{1}\right)} \psi(\alpha) d \alpha, j \in[2 . . M]
$$

the gradient vector and Hessian matrix of the objective take the respective forms

$$
\begin{aligned}
\nabla g_{j}\left(T_{1}\right)= & \left(b_{1}^{i_{j}}-b_{1}^{i_{j-1}}\right) \int_{0}^{\alpha_{i_{j-1}}\left(T_{1}\right)} \psi(\alpha) d \alpha+\nabla \alpha_{i_{j-1}}\left(T_{1}\right)\left\langle b_{1}^{i_{j}}-b_{1}^{i_{j-1}}, T_{1}\right\rangle \psi\left(\alpha_{i_{j-1}}\left(T_{1}\right)\right), \\
\nabla^{2} g_{j}\left(T_{1}\right)= & 2 \frac{\left(b_{1}^{i_{j}}-b_{1}^{i_{j-1}}\right)^{t}\left(b_{1}^{i_{j}}-b_{1}^{i_{j-1}}\right)}{G_{i_{j}}-G_{i_{j-1}}} \psi\left(\alpha_{i_{j-1}}\left(T_{1}\right)\right) \\
& +\nabla \alpha_{i_{j-1}}\left(T_{1}\right)\left(\nabla \alpha_{i_{j-1}}\left(T_{1}\right)\right)^{t}\left\langle b_{1}^{i_{j-1}}-b_{1}^{i_{j}}, T_{1}\right\rangle \psi^{\prime}\left(\alpha_{i_{j-1}}\left(T_{1}\right)\right) \\
= & \frac{\left(b_{1}^{i_{j}}-b_{1}^{i_{j-1}}\right)^{t}\left(b_{1}^{i_{j}}-b_{1}^{i_{j-1}}\right)}{G_{i_{j}}-G_{i_{j-1}}}\left[2 \psi\left(\alpha_{i_{j-1}}\left(T_{1}\right)\right)+\frac{\left\langle b_{1}^{i_{j-1}}-b_{1}^{i_{j}}, T_{1}\right\rangle \psi^{\prime}\left(\alpha_{i_{j-1}}\left(T_{1}\right)\right)}{G_{i_{j}}-G_{i_{j-1}}}\right] .
\end{aligned}
$$

A sufficient condition for the concavity of the profit function is that, for every index $j$, the matrix $\nabla^{2} g_{j}\left(T_{1}\right)$ be negative semidefinite. This condition will be satisfied if either 
1. The density function $\psi$ is uniform over the interval $\left[0, \alpha_{\max }\right]$, i.e.,

$$
\psi= \begin{cases}\frac{1}{\alpha_{\max }} & \text { if } \alpha \in\left[0, \alpha_{\max }\right] \\ 0 & \text { otherwise }\end{cases}
$$

2. $\psi(\alpha)$ is nondecreasing and $\left\langle b_{1}^{i_{j-1}}-b_{1}^{i_{j}}, T_{1}\right\rangle<0$ for $j \in[2 . . M]$ and $T_{1} \in \Lambda$.

Since $\alpha_{i_{j}}$ is an affine function of $T_{1}$, the profit function $f\left(T_{1}\right)$ is piecewise concave whenever one of the above conditions holds. If the market structure is known, i.e., $R^{\left|A_{1}\right|}=\bigcup_{i} \Lambda_{i}$, the optimal price schedule can be obtained by solving a finite set of mathematical programs:

$$
\max _{i} \max _{T_{1} \in \Lambda_{i}} f_{\Lambda_{i}}\left(T_{1}\right)
$$

where, in the uniform case, the objective functions take the form

$$
f_{\Lambda_{i}}\left(T_{1}\right)=\frac{1}{\alpha_{\max }}\left\{\left\langle b_{1}^{i_{1}}-b_{1}^{i_{2}}, T_{1}\right\rangle \alpha_{i_{1}}+\left\langle b_{1}^{i_{2}}-b_{1}^{i_{3}}, T_{1}\right\rangle \alpha_{i_{2}}+\ldots+\left\langle b_{1}^{i_{M-1}}-b_{1}^{i_{M}}, T_{1}\right\rangle \alpha_{i_{M-1}}\right\}+\left\langle b_{1}^{i_{M}}, T_{1}\right\rangle .
$$

However, by adding the equalities

$$
\begin{aligned}
\left\langle b_{1}^{i_{1}}-b_{1}^{i_{2}}, T_{1}\right\rangle & =\left(G_{i_{2}}-G_{i_{1}}\right) \alpha_{i_{1}}-\left\langle b_{2}^{i_{1}}-b_{2}^{i_{2}}, T_{2}\right\rangle \\
\left\langle b_{1}^{i_{2}}-b_{1}^{i_{3}}, T_{1}\right\rangle & =\left(G_{i_{3}}-G_{i_{2}}\right) \alpha_{i_{2}}-\left\langle b_{2}^{i_{2}}-b_{2}^{i_{3}}, T_{2}\right\rangle \\
& \vdots \\
\left\langle b_{1}^{i_{M-1}}-b_{1}^{i_{M}}, T_{1}\right\rangle & =\left(G_{i_{M}}-G_{i_{M-1}}\right) \alpha_{i_{M-1}}-\left\langle b_{2}^{i_{M-1}}-b_{2}^{i_{M}}, T_{2}\right\rangle .
\end{aligned}
$$

one obtains $\left\langle b_{1}^{i_{M}}, T_{1}\right\rangle=\left\langle b_{1}^{i_{1}}, T_{1}\right\rangle+\left\langle b_{2}^{i_{1}}-b_{2}^{i_{M}}, T_{2}\right\rangle-\left[\left(G_{i_{2}}-G_{i_{1}}\right) \alpha_{i_{1}}+\ldots+\left(G_{i_{M}}-G_{i_{M-1}}\right) \alpha_{i_{M-1}}\right]$ and, finally, the quadratic expression

$$
\begin{aligned}
f_{\Lambda_{i}}\left(T_{1}\right)= & \frac{1}{\alpha_{\max }}\left\{\left(G_{i_{2}}-G_{i_{1}}\right)\left(\alpha_{i_{1}}-\frac{\alpha_{\max }+\frac{\left\langle b_{2}^{i_{1}}-b_{2}^{i_{2}}, T_{2}\right\rangle}{G_{i_{2}}-G_{i_{1}}}}{2}\right)^{2}+\ldots\right. \\
& +\left(G_{i_{M}}-G_{i_{M-1}}\right)\left(\alpha_{i_{M-1}}-\frac{\alpha_{\max }+\frac{\left\langle b_{2}^{i_{M-1}}-b_{2}^{i_{M}}, T_{2}\right\rangle}{G_{i_{M}}-G_{i_{M-1}}}}{2}\right)^{2} \\
& \left.-\frac{1}{4} \sum_{j=1}^{M-1}\left(G_{i_{j+1}}-G_{i_{j}}\right)\left(\alpha_{\max }+\frac{\left\langle b_{2}^{i_{j}}-b_{2}^{i_{j+1}}, T_{2}\right\rangle}{G_{i_{j+1}}-G_{i_{j}}}\right)^{2}\right\} \\
& +\left\langle b_{1}^{i_{1}}, T_{1}\right\rangle+\left\langle b_{2}^{i_{1}}-b_{2}^{i_{M}}, T_{2}\right\rangle .
\end{aligned}
$$

Hence the functions $f_{\Lambda_{i}}\left(T_{1}\right)$ are differentiable concave quadratic functions that can be maximized by the steepest ascent algorithm or, on networks of moderate sizes, is amenable to conjugate gradient or quasi-Newton methods. 


\section{Back to the Air Canada example}

In the introductory example of Section 2, there are three flights (paths) with respective generalized costs:

$$
\begin{aligned}
& p_{1}=1320+36 \alpha \\
& p_{2}=T_{M V}+720+26 \alpha \\
& p_{3}=T_{M V}+T_{V S}+18 \alpha .
\end{aligned}
$$

Seven market structures correspond to seven regions $\left\{\Lambda_{i}\right\}_{i=0}^{6}$ in the Euclidian plane (see Figure 3). We now proceed to analyze each region, assuming that total demand is equal to 1000 passengers and that the density function is uniform with maximal VOT equal to $\alpha_{\max }=90$. Calculations, albeit straightforward, are tedious.

- Region 0 (Main region)

This corresponds to the market structure where all services (products, flights) are competitive, i.e., in the interior of its region, all flights carry positive flow. It is described as

$$
\Lambda_{0}=\left\{T_{M V} \geq 0, T_{V S} \geq 0: 600 \leq T_{M V}, 4 T_{M V}-5 T_{V S}+1200 \leq 0, T_{V S} \leq 1440\right\}
$$

The profit function in this area is

$$
f_{\Lambda_{0}}\left(T_{1}\right)=1000 \frac{1}{3600}\left(-4 T_{M V}^{2}+6000 T_{M V}-5 T_{V S}^{2}+7200 T_{V S}\right),
$$

whose maximum $f^{*} \approx \$ 1334000$ is achieved when $T_{M V}^{*}=\$ 683$ and $T_{V S}^{*}=\$ 787$.

- Region 1: $\Lambda_{1}=\left\{T_{M V} \geq 0, T_{V S} \geq 0: 600 \leq T_{M V} \leq 1500, T_{V S} \geq 1440\right\}, h_{p_{3}}=0$

- profit function: $f_{\Lambda_{1}}\left(T_{1}\right)=\frac{10}{9}\left(-T_{M V}^{2}+1500 T_{M V}\right)$

- optimal profit: $f^{*}=\$ 625000$

- optimal solution: $T_{M V}^{*}=\$ 750 \quad T_{V S}^{*} \geq \$ 1440$

- Region 2: $\Lambda_{2}=\left\{T_{M V} \geq 0, T_{V S} \geq 0: T_{M V} \leq 600, T_{V S} \geq 1440\right\}, h_{p_{1}}=h_{p_{3}}=0$

- profit function: $f_{\Lambda_{2}}\left(T_{1}\right)=1000 T_{M V}$

- optimal profit: $f^{*}=\$ 600000$ 
- optimal solution: $T_{M V}^{*}=\$ 600 \quad T_{V S}^{*} \geq \$ 1440$

- Region 3: $\Lambda_{3}=\left\{T_{M V} \geq 0, T_{V S} \geq 0: T_{M V} \leq 600,720 \leq T_{V S} \leq 1400\right\}, h_{p_{1}}=0$

- profit function: $f_{\Lambda_{3}}\left(T_{1}\right)=1000 \times \frac{1}{720}\left(-T_{M V}^{2}+1440 T_{V S}\right)+T_{M V}$

- optimal profit: $f^{*}=\$ 1320000$

- optimal solution: $T_{M V}^{*}=\$ 600 \quad T_{V S}^{*}=\$ 720$

- Region 4: $\Lambda_{4}=\left\{T_{M V} \geq 0, T_{V S} \geq 0: T_{M V}+T_{V S} \leq 1320, T_{V S} \leq 720\right\}, h_{p_{1}}=h_{p_{2}}=0$

- profit function: $f_{\Lambda_{4}}\left(T_{1}\right)=1000\left(T_{M V}+T_{V S}\right)$,

- optimal profit: $f^{*}=\$ 1320000$

- optimal solution: Any combination in $\Lambda_{4}$ such that $T_{M V}^{*}+T_{V S}^{*}=\$ 1320$

- Region 5: $\Lambda_{5}=\left\{T_{M V} \geq 0, T_{V S} \geq 0: 1320 \leq T_{M V}+T_{V S} \leq 2940,4 T_{M V}-5 T_{V S}+1200 \leq\right.$ $0\}, h_{p_{2}}=0$

- profit function: $f_{\Lambda_{5}}\left(T_{1}\right)=1000 \frac{1}{1620}\left(-\left(T_{M V}+T_{V S}\right)^{2}+2940\left(T_{M V}+T_{V S}\right)\right)$

- optimal profit: $f^{*} \approx \$ 1334000$

- optimal solution: Any combination in $\Lambda_{5}$ such that $T_{M V}^{*}+T_{V S}^{*}=\$ 1470$

- Region 6: $\Lambda_{6}=\left\{T_{M V} \geq 0, T_{V S} \geq 0: T_{M V}+T_{V S} \geq 2940, T_{M V} \geq 1500\right\}, h_{p_{2}}=h_{p_{3}}=0$

- profit function: $f_{\Lambda_{6}}\left(T_{1}\right)=0$

- optimal profit: $f^{*}=\$ 0$

- optimal solution: Any combination of fares that belongs to $\Lambda_{6}$

It is interesting to take a closer look at some regions. For instance, in regions 2, 3 and 4, the leader company's combination of fare and time dominates all competing proposals, and this results in Air Canada attracting all consumers to its two-leg flight. At the other end of the spectrum, Air Canada's fares in region 6 are so high that they fail to attract even passengers whose value of time is at its maximum $\alpha_{\max }$. In areas 0,1 and 5 , the fares set by the leader company achieve a trade-off between the number of passengers attracted and their willingness to pay. 
The optimum is achieved in region 5. Since path 2 is dominated, the corresponding flow is zero, and only the sum of the fares $T_{M V}+T_{V S}$ on Air Canada's two-leg flight matters. The trade-off VOT at which passengers are indifferent between path $p_{1}$ and $p_{3}$ is the solution of the linear equation

$$
1320+36 \alpha=1470+18 \alpha
$$

i.e., $\alpha^{*}=25 / 3$. The market share for Air Canada's two-leg flight is equal to the probability that a passenger's VOT be larger than $\alpha^{*}$, i.e.,

$$
\int_{\alpha^{*}}^{90} \psi(\alpha) d \alpha=1-\frac{25}{3 \times 90} \approx .907
$$

Therefore, at the optimum, 907 passengers fly Air Canada between Montreal and Shanghai.

\section{Conclusion}

In this paper, we have developed a bilevel pricing model for a service firm. Two key features of the model is that it explicitly takes into account customer behavior (thus allowing room for price discrimination) as well as the underlying network topology of the problem.

While, for ease of presentation, we restricted ourselves to a simple form of the model, its formulation can easily be extended to deal with more refined descriptions of user behavior, involving for instance more than two criteria, and to incorporate features such as: finite capacities, upper bounds on decision variables, more general functional forms of the cost functions, etc. A more ambitious goal is to integrate the current model within a dynamic framework, thus closing the gap between the bilevel pricing model and more traditional "yield management" approaches adopted in the airline industry.

Finally, a word about algorithmic complexity. From the computational point of view, our model looks complex, as it requires the solution of a large (exponential) number of subproblems. However our numerical experiences tend to show that, when started in a region which corresponds to flows that are (almost) all positive, convergence towards an optimal or near-optimal solution is frequently achieved. We also investigated successfully a discretization approach where the densities $\psi_{w}$ are replaced by probability mass functions. The resulting approximation is then reformulated as a large mixed integer program (see Côté et al. 2002) that can be tackled either by commercial codes or heuristic procedures. 


\section{Acknowledgments}

This research has been supported by NSERC (Canada), MITACS and FQRNT (Québec).

\section{References}

Anderson, E. J., P. Nash. 1987. Linear Programming in Infinite-Dimensional Spaces.. John Wiley and Sons, New York, NY.

Belobaba, P.P. 1987. Airline yield management: An overview of seat inventory control. Transportation Science $\mathbf{2 1}$ 63-73.

Côté, J.-P., P. Marcotte, G. Savard. 2002. A Bilevel Modeling Approach to Pricing and Fare Optimization in the Airline Industry. GERAD Research Report G-2002-71, Montreal, Canada.

Gabszewicz, J., J.-F. Thisse. 1979a. On the Nature of Competition with Differentiated Products. The Economic Journal 96 160-172.

Gabszewicz, J., J.-F. Thisse. 1979b. Price competition, quality and income disparities. Journal of Economic Theory 20 340-359.

Hotelling, H. 1929. Stability in competition. Economic Journal 39 41-57.

Krouse, C.K. 1990. Theory of Industrial Economics. Basil Blackwell, Oxford, UK.

Labbé, M., P. Marcotte, G. Savard. 1998. A bilevel model of taxation and its application to optimal highway pricing. Management Science 44 1595-1607.

Leurent, F. 1993. Cost versus time equilibrium over a network. European Journal of Operational Research 71 205-221.

Loridan, P., J. Morgan. 1996. Weak via strong Stackelberg problem: new results. Journal of Global Optimization 8 263-287.

P. Marcotte. Reformulations of a bicriterion equilibrium problem. In: Reformulation Nonsmooth, Piecewise Smooth, Semismooth and Smoothing Methods, Masao Fukushima and Liqun Qi eds., Kluwer, Dordrecht (1999) 269-292.

Marcotte, P., D.L. Zhu. 1997. Equilibria with infinitely many differentiated classes of customers. J.-S. Pang, M. Ferris, eds. Complementarity and Variational Problems, State of Art, SIAM, Philadelphia, USA. 234-258. 
Martin, S. 1993. Advanced Industrial Economics, Basil Blackwell, Oxford, UK.

McGill, J.L., G.J. Van Ryzin. 1999. Revenue Management: Research Overview and Prospects. Transportation Science 33 233-256.

Phlips, L. 1983. The Economics of Price Discrimination. Cambridge University Press, Cambridge, UK. 
Phlips, L., J.-F. Thisse. 1982. Spatial competition and the theory of differential markets. Journal of Industrial Economics 31 1-9. 